\title{
EVALUACIÓN FORMATIVA Y COMPARTIDA EN EDUCACIÓN PRIMARIA. UNA EXPERIENCIA DE AUTOEVALUACIÓN Y AUTOCALIFICACIÓN EN EL ÁREA dE MATEMÁTICAS
}

Training and shared evaluation in primary education. An experience of self-assessment and self-qualification in the mathematics area

\section{Avaliação formativa e partilhada na educação primária. Uma experiência de autoavaliação e autoclassificação na área da matemática}

\section{Marcos Herranz Sancho (1)}

(1) Junta Castilla y León, España. Teléfono: 659595790. Correo electrónico: marcosancho26@gmail.com

\section{Resumen}

El objeto principal de este estudio es realizar una experiencia de evaluación formativa y compartida en la etapa de Educación Primaria en el área de Matemáticas. El diseño de investigación ha sido cualitativo y se ha realizado mediante un estudio de caso longitudinal a lo largo de dos trimestres escolares con los mismos alumnos. Hemos utilizado las técnicas de la autoevaluación, la evaluación compartida, la autocalificación y la calificación dialogada entre los alumnos y el profesor. Los resultados nos han mostrado que es viable poner en funcionamiento esta metodología de evaluación y calificación con alumnos de Educación Primaria, que se producen aprendizajes en todas las personas implicadas en los procesos de evaluación y que mejora el clima de clase como consecuencia de la participación de los alumnos.

Palabras clave: Evaluación Formativa; Autoevaluación; Autocalificación

\begin{abstract}
The main purpose of this study is to carry out a formative and shared evaluation experiment in the stage of Primary Education in Mathematics. The research methodology has been qualitative and has been carried out through a longitudinal case study over two school trimesters with the same students. We have used the techniques of self-assessment, shared assessment, self-rating and the dialogue between the students and the teacher. The results show that it is viable to put this methodology of evaluation
\end{abstract}


and qualification into operation with primary education students, that learning takes place in all the people involved in the evaluation processes and that the classroom environment improves as a result of the participation of the students.

Keywords: Formative Evaluation; Self-assessment; Self-rating

\section{Resumo}

O objetivo principal deste estudo é realizar uma experiência de avaliação formativa e partilhada na Educação Primária na área da Matemática. Usou-se um desenho de investigação qualitativo, através de um estudo de caso longitudinal, com os mesmos alunos, ao longo de dois trimestres escolares. Utilizámos as técnicas de autoavaliação, avaliação partilhada, autoclassificação e a classificação dialogada entre alunos e professor. Os resultados mostraram-nos que é possível pôr em prática esta metodologia de avaliação e classificação com alunos do ensino primário, que ocorre aprendizagem em todas as pessoas envolvidas nos processos de avaliação e que melhora o clima na aula como consequência da participação dos alunos.

Palavras-chave: Avaliação formativa; Autoavaliação; Autoclassificação

\section{Introducción}

A lo largo de esta experiencia mostramos la forma en la que desarrollamos nuestro sistema de evaluación. Con ello pretendemos que la evaluación se convierta en una actividad de aprendizaje (Álvarez Méndez, 2001) y que la información que proporcionan los instrumentos de evaluación sea utilizada para generar nuevos aprendizajes. Estos se producirán en todas las personas implicadas en los procesos de evaluación, tanto alumnos como profesores, así como en el propio proceso de enseñanza y aprendizaje, (López, 2006). De esta forma realizamos una auténtica evaluación formativa.

Los principales instrumentos de evaluación que utilizamos son: los cuestionarios de autoevaluación, las fichas de autocalificación, la ficha de registro de observación grupal, y las entrevistas individuales entre el profesor y los alumnos.

\section{Contextualización}

La experiencia se ha llevado a cabo en un centro público de la provincia de Segovia de septiembre de 2018 a abril de 2019. Es un centro de una línea completa, con una ratio 
media de 15 alumnos por aula. El nivel socioeconómico y cultural de las familias pertenecientes a la comunidad educativa es medio-alto.

La asignatura en la que he realizado nuestra experiencia es la de Matemáticas en el Tercer curso de Educación Primaria. Esta asignatura se imparte cinco horas semanales en sesiones de una hora cada día. El grupo clase lo componen doce alumnos entre los que no hay ningún Alumno Con Necesidades Específicas de Apoyo Educativo.

\section{Diseño y desarrollo}

El diseño de investigación corresponde con un estudio de caso longitudinal (dos trimestres escolares completos con los mismos alumnos) en una situación concreta (Stake, 1998). Está dentro del paradigma naturalista (Guba, 1989). Utilizamos una metodología de investigación predominantemente cualitativa al recoger una gran cantidad y variedad de datos (Rodríguez, Gil \& García, 1996).

Las actividades de aprendizaje que hemos llevado a cabo han sido: actividades de cálculo mental, de asimilación de conceptos, de operatoria y de resolución de problemas.

Los instrumentos de evaluación que utilizamos son: diario del profesor, ficha de registro de observación grupal, fichas y cuadernos de trabajo de los alumnos, cuestionarios de autoevaluación, fichas de autocalificación y entrevistas individuales entre el profesor y los alumnos.

El alumnado recibe feedback en varios momentos del proceso de enseñanza y aprendizaje: en la evaluación inicial, por el profesor y por otros compañeros; en las dinámicas de clase, por el profesor; (preguntas, dudas, aclaraciones...) en dinámicas grupales y cooperativas, de los propios compañeros; en los procesos de coevaluación de los trabajos, de los compañeros; y, en los procesos de evaluación compartida, del profesor.

Los alumnos llegan a la calificación mediante la autocalificación y la calificación dialogada con el profesor. Para ello empleamos un sistema de escala graduada con una serie de criterios para obtener las diferentes calificaciones que es conocido por los alumnos.

Para llegar a la calificación final del trimestre los alumnos han tenido en su poder los cuestionarios de autoevaluación de cada unidad didáctica realizada a lo largo del trimestre. Posteriormente se ha realizado la calificación dialogada con el profesor 
mediante una entrevista individual en la que se tendría que llegar a un acuerdo. En caso de no haber acuerdo se dará participación al grupo-clase.

Los resultados de la evolución del rendimiento académico de los alumnos durante los dos trimestres en los que se ha desarrollado la experiencia han sido los siguientes:

Tabla 1.

Rendimiento académico de los alumnos. Resultados en números, porcentajes y calificación media.

\begin{tabular}{|c|c|c|c|}
\hline & $\begin{array}{c}\text { Primer trimestre. } \\
\text { Calificación media: } \\
2,83 \\
\end{array}$ & $\begin{array}{c}\text { Segundo trimestre. } \\
\text { Calificación media: } \\
3,08 \\
\end{array}$ & $\begin{array}{c}\text { Tercer trimestre. } \\
\text { Calificación media: } \\
--\end{array}$ \\
\hline Sobresalientes & $4(33,33 \%)$ & $4(33,33 \%)$ & -- \\
\hline Notables & $4(33,33 \%)$ & $5(41,66 \%)$ & -- \\
\hline Bienes & $2(16,66 \%)$ & $2(16,66 \%)$ & -- \\
\hline Suficientes & $2(16,66 \%)$ & $1(8,33 \%)$ & -- \\
\hline Insuficientes & $0(0 \%)$ & $0(0 \%)$ & -- \\
\hline TOTALES & $12(100 \%)$ & $12 / 100 \%)$ & -- \\
\hline
\end{tabular}

Escala: Sobresaliente $=4$. Notable $=3$. Bien $=2$. Suficiente $=1$. Insuficiente $=0$.

El primer dato que consideramos destacable es que todos los alumnos superan la asignatura. Las calificaciones altas (sobresaliente y notable) pasan de ser el 66,66\% en la primera evaluación a ser el 74,99\% en la segunda. Las calificaciones bajas (aprobado y bien) pasan del 31,32\% al 24,99\%. La nota media del trimestre pasa a ser de 2,83 en la primera evaluación a 3,08 en la segunda. Ha habido tres alumnos que han aumentado el nivel de su calificación y un alumno que ha bajado dicho nivel.

Los alumnos han sabido interpretar la información que han aportado los instrumentos de evaluación y utilizarla para generar más aprendizaje. También ha servido para justificar con más rigor sus autocalificaciones.

Así el/la alumno/a P3101819 justifica su calificación de la segunda evaluación de la siguiente manera:

"He ayudado a los compañeros, me sé muy bien las tablas y he sabido hacer bien los problemas".

El/la alumno/a P3021819 justifica su calificación de la segunda evaluación de la siguiente manera:

"Hago las actividades de clase, a veces no termino a tiempo y me tienen que ayudar para entenderlo".

\section{Evaluación}

Las principales ventajas encontradas han sido las siguientes:

-Los alumnos se centran en los procesos y no en los resultados.

Evaluación formativa y compartida en Educación Primaria. Una experiencia de autoevaluación y autocalificación en el área de Matemáticas 
-Los alumnos aprovechan los instrumentos de evaluación para obtener información en torno a sus aprendizajes.

-Los alumnos han desarrollado una gran capacidad de razonar, argumentar y justificar sus evaluaciones y calificaciones.

-Se ha conseguido un gran clima de aula al tener los alumnos unos referentes claros para su evaluación y calificación.

-Se ha producido una constante mejora en el desarrollo profesional del maestro.

-Se ha producido mejoras en el rendimiento académico de los alumnos a lo largo de los trimestres.

Entre los inconvenientes que han surgido destacamos:

-El trabajo inicial es grande para preparar la diversidad de instrumentos.

-Hay que explicar de forma completa y detallada de método de evaluación y calificación.

-En ocasiones algunos alumnos tienen dificultades para utilizar los instrumentos de evaluación y calificación.

\section{Conclusiones}

Las conclusiones más destacadas han sido las siguientes:

-Es posible llevarlo a cabo en las condiciones de trabajo normales de un maestro de Educación Primaria.

-Es un sistema que fomenta aprendizajes en todas las personas que intervienen en el mismo.

-Se fomenta la participación de los alumnos.

-Se ha mejorado el rendimiento académico de los alumnos como consecuencia de la mejora de los aprendizajes.

\section{Referencias}

Álvarez Méndez, J. M. (2001). Evaluar para conocer, examinar para excluir. Madrid: Morata.

Guba, E. G. (1989). Criterios de credibilidad en la investigación naturalista. En J. Gimeno Sacristán \& A. Pérez Gómez. (Eds.), La enseñanza: su teoría y su práctica (pp. 148-165). Madrid: Akal. 
López Pastor, V. M. (Coord.) (2006). La evaluación en educación Física. Revisión de los modelos tradicionales y planteamiento de una alternativa: la evaluación formativa y compartida. Madrid: Miño y Dávila.

Rodríguez Gómez, G., Gil Flores, J., \& García Jiménez, E. (1996). Metodología de la investigación cualitativa. Archidona, Málaga: Aljibe.

Stake, R. E. (1998). Investigación con estudio de casos. Madrid: Morata. 